PROCEEDINGS OF THE

AMERICAN MATHEMATICAL SOCIETY

Volume 134, Number 8, Pages 2179-2187

S 0002-9939(06)08224-4

Article electronically published on March 14, 2006

\title{
A SHORT PROOF OF THE ZEILBERGER-BRESSOUD $q$-DYSON THEOREM
}

\author{
IRA M. GESSEL AND GUOCE XIN
}

(Communicated by John R. Stembridge)

\begin{abstract}
We give a formal Laurent series proof of Andrews's $q$-Dyson Conjecture, first proved by Zeilberger and Bressoud.
\end{abstract}

\section{INTRODUCTION}

Freeman Dyson, motivated by a problem in particle physics, conjectured the following identity in 1962:

Theorem 1.1 (Dyson's Conjecture). For nonnegative integers $a_{0}, a_{1}, \ldots, a_{n}$,

$$
\mathrm{CT} \prod_{0 \leq i \neq j \leq n}\left(1-\frac{x_{i}}{x_{j}}\right)^{a_{j}}=\frac{\left(a_{0}+a_{1}+\cdots+a_{n}\right) !}{a_{0} ! a_{1} ! \cdots a_{n} !},
$$

where $\mathrm{CT}_{\mathbf{x}}$ denotes the constant term in $x_{0}, \ldots, x_{n}$.

Dyson's conjecture was quickly proved by Wilson [12 and independently by Gunson [6]. An elegant recursive proof was published by Good [5] in 1970.

A $q$-analog of Theorem 1.1 was conjectured by George Andrews [1 in 1975:

Theorem 1.2 (Zeilberger-Bressoud). For any nonnegative integers $a_{0}, a_{1}, \ldots, a_{n}$,

$$
\mathrm{CT} \prod_{\mathbf{x}}\left(\frac{x_{i}}{x_{j}}\right)_{a_{i}}\left(\frac{x_{j}}{x_{i}} q\right)_{a_{j}}=\frac{(q)_{a_{0}+\cdots+a_{n}}}{(q)_{a_{0}} \cdots(q)_{a_{n}}},
$$

where $(z)_{m}=(1-z)(1-q z) \cdots\left(1-q^{m-1} z\right)$.

Andrews's $q$-Dyson conjecture attracted much interest, but was not proved until 1985, in a combinatorial tour de force by Zeilberger and Bressoud [16]. In related work, Stanley [9, 10] reformulated the conjecture in terms of symmetric functions and proved a limiting form of the conjecture, and Kadell [7] proved the 4-variable case using an approach similar to Good's. Bressoud and Goulden 3] extended the Zeilberger-Bressoud approach to some generalizations of (1.2), and Stembridge [1] gave an elegant recursive proof of the equal parameter case. Cherednik [4] proved the Macdonald constant term conjecture for root systems 8, which generalizes the equal parameter case.

Received by the editors December 21, 2004 and, in revised form, February 12, 2005. 2000 Mathematics Subject Classification. Primary 05A30; Secondary 33D70.

Key words and phrases. $q$-series, Dyson's conjecture, Laurent series, partial fractions.

The first author was partially supported by NSF Grant DMS-0200596.

(C)2006 American Mathematical Society Reverts to public domain 28 years from publication 
Zeilberger and Bressoud's combinatorial proof is, so far, the only proof of Andrews's $q$-Dyson conjecture. Here we give a very different and shorter proof, using properties of the formal Laurent series.

The idea behind the proof is the well-known fact that to prove the equality of two polynomials of degree at most $d$, it is sufficient to prove that they are equal at $d+1$ points. As is often the case, points at which the polynomials vanish are most easily dealt with.

It is not difficult to show that for fixed nonnegative integers $a_{1}, \ldots, a_{n}$, both sides of (1.2) are polynomials in $q^{a_{0}}$ of degree at most $a_{1}+\cdots+a_{n}$ and that the polynomial corresponding to the right side of (1.2) vanishes for $a_{0}=-1,-2, \ldots,-\left(a_{1}+\cdots+a_{n}\right)$. The main part of our proof is to show that the polynomial corresponding to the left-hand side of (1.2) also vanishes at these points. We do this by expanding the left-hand side in partial fractions in such a way that we can show that each summand has zero constant term. The proof is completed by observing that the case $a_{0}=0$ of (1.2) is equivalent to the $n$-variable case.

\section{BASIC FACTS}

We use the following standard notation:

$$
(z)_{n}=\frac{(z)_{\infty}}{\left(z q^{n}\right)_{\infty}}=\prod_{m=0}^{\infty} \frac{\left(1-z q^{m}\right)}{\left(1-z q^{m+n}\right)} .
$$

Note that if $p$ is a nonnegative integer, then

$$
\begin{aligned}
(z)_{p} & =(1-z)(1-z q) \cdots\left(1-z q^{p-1}\right), \\
(z)_{-p} & =\frac{1}{\left(1-z q^{-1}\right)\left(1-z q^{-2}\right) \cdots\left(1-z q^{-p}\right)} .
\end{aligned}
$$

The $q$-binomial coefficients are defined for all integers $n$ and nonnegative integers $m$ by

$$
\left[\begin{array}{c}
n \\
m
\end{array}\right]=\frac{\left(q^{n-m+1}\right)_{m}}{(q)_{m}}=\frac{\left(1-q^{n}\right)\left(1-q^{n-1}\right) \cdots\left(1-q^{n-m+1}\right)}{(1-q)\left(1-q^{2}\right) \cdots\left(1-q^{m}\right)} .
$$

For $0 \leq m \leq n$, we have

$$
\left[\begin{array}{c}
n \\
m
\end{array}\right]=\frac{(q)_{n}}{(q)_{m}(q)_{n-m}}
$$

The well-known $q$-binomial theorem [2, Theorem 2.1] is the identity

$$
\frac{(a z)_{\infty}}{(z)_{\infty}}=\sum_{k=0}^{\infty} \frac{(a)_{k}}{(q)_{k}} z^{k}
$$

Setting $z=u q^{n}$ and $a=q^{-n}$ in (2.4), and using the definition (2.1), we obtain

$$
(u)_{n}=\sum_{k=0}^{\infty} q^{k(k-1) / 2}\left[\begin{array}{l}
n \\
k
\end{array}\right](-u)^{k}
$$

for all integers $n$.

We will also need the easily-proved identity

$$
\left(\frac{x_{i}}{x_{j}}\right)_{l}\left(\frac{x_{j}}{x_{i}} q\right)_{m}=q^{\left(\begin{array}{c}
m+1 \\
2
\end{array}\right)}\left(-\frac{x_{j}}{x_{i}}\right)^{m}\left(\frac{x_{i}}{x_{j}} q^{-m}\right)_{l+m} .
$$




\section{THE PROOF}

Let us fix $\mathbf{a}=\left(a_{1}, \ldots, a_{n}\right)$, where $a_{1}, \ldots, a_{n}$ are nonnegative integers. Let $a=$ $a_{1}+\cdots+a_{n}$ and let

$$
P_{\mathbf{a}}\left(q^{b}\right)=\frac{(q)_{a}}{(q)_{a_{1}} \cdots(q)_{a_{n}}}\left[\begin{array}{c}
b+a \\
a
\end{array}\right]=\frac{\left(1-q^{b+a}\right)\left(1-q^{b+a-1}\right) \cdots\left(1-q^{b+1}\right)}{(q)_{a_{1}} \cdots(q)_{a_{n}}} .
$$

Then the right-hand side of (1.2) is equal to $P_{\mathbf{a}}\left(q^{a_{0}}\right)$. We observe that

(i) $P_{\mathbf{a}}\left(q^{b}\right)$ is a polynomial in $q^{b}$ of degree at most $a$.

(ii) $P_{\mathbf{a}}\left(q^{b}\right)=0$ for $q^{b}=q^{-1}, q^{-2}, \ldots, q^{-a}$.

Moreover, $P_{\mathbf{a}}\left(q^{b}\right)$ is uniquely determined by these two properties up to a constant factor (which may depend on $q$ but not on $b$ ).

Let $Q_{\mathbf{a}}\left(q^{b}\right)$ be defined by

$$
Q_{\mathbf{a}}\left(q^{b}\right)=\mathrm{CT}_{\mathbf{x}} \prod_{j=1}^{n}\left(\frac{x_{0}}{x_{j}}\right)_{b}\left(\frac{x_{j}}{x_{0}} q\right)_{a_{j}} \prod_{1 \leq i<j \leq n}\left(\frac{x_{i}}{x_{j}}\right)_{a_{i}}\left(\frac{x_{j}}{x_{i}} q\right)_{a_{j}} .
$$

Then the left-hand side of (1.2) equals $Q_{\mathbf{a}}\left(q^{a_{0}}\right)$.

In fact $Q_{\mathbf{a}}\left(q^{b}\right)$ is well defined for negative integers $b$ if we treat the rational function in (3.2) as a Laurent series in $x_{0}$. The following two lemmas show that $Q_{\mathbf{a}}\left(q^{b}\right)$ equals $P_{\mathbf{a}}\left(q^{b}\right)$ up to a constant multiple.

Lemma 3.1. For fixed $\mathbf{a} \in \mathbb{N}^{n}, Q_{\mathbf{a}}\left(q^{b}\right)$ is a polynomial in $q^{b}$ of degree at most $a$.

Lemma 3.2 (Main Lemma). For any $\mathbf{a} \in \mathbb{N}^{n}, Q_{\mathbf{a}}\left(q^{b}\right)=0$ for $b=-1,-2, \ldots,-a$.

Lemma 3.2 is the heart of our proof of Theorem 1.2. We will prove it in Sections 4 and 5. Here we give the rest of the proof of Theorem 1.2 .

Proof of Lemma 3.1, By (2.6) we have

$$
\left(\frac{x_{0}}{x_{j}}\right)_{b}\left(\frac{x_{j}}{x_{0}} q\right)_{a_{j}}=q^{\left({ }^{a_{j}+1}\right)}\left(-\frac{x_{j}}{x_{0}}\right)^{a_{j}}\left(\frac{x_{0}}{x_{j}} q^{-a_{j}}\right)_{b+a_{j}},
$$

for all integers $b$, where both sides are regarded as Laurent series in $x_{0}$.

Thus $Q_{\mathbf{a}}\left(q^{b}\right)$ can be rewritten as

$$
\left.\underset{\mathbf{x}}{\mathrm{CT}} L\left(x_{1}, \ldots, x_{n}, \mathbf{a}\right) \prod_{j=1}^{n} q^{\left(a_{j}+1\right.}\right)\left(-\frac{x_{j}}{x_{0}}\right)^{a_{j}}\left(\frac{x_{0}}{x_{j}} q^{-a_{j}}\right)_{b+a_{j}},
$$

where $L\left(x_{1}, \ldots, x_{n}, \mathbf{a}\right)$ is a Laurent polynomial in $x_{1}, \ldots, x_{n}$ independent of $x_{0}$ and $b$.

Using the $q$-binomial theorem (2.5), we see that for $1 \leq j \leq n$,

$$
\left.q^{\left(a_{j}+1\right.}\right)\left(-\frac{x_{j}}{x_{0}}\right)^{a_{j}}\left(\frac{x_{0}}{x_{j}} q^{-a_{j}}\right)_{b+a_{j}}=\sum_{k_{j} \geq 0} C\left(k_{j}\right)\left[\begin{array}{c}
b+a_{j} \\
k_{j}
\end{array}\right] x_{0}^{k_{j}-a_{j}} x_{j}^{a_{j}-k_{j}},
$$

where $C\left(k_{j}\right)=(-1)^{k_{j}} q^{\left(\begin{array}{c}a_{j}+1 \\ 2\end{array}\right)+\left(\begin{array}{c}k_{j} \\ 2\end{array}\right)-k_{j} a_{j}}$.

Expanding the product in (3.3) and taking the constant term in $x_{0}$, we get

$$
Q_{\mathbf{a}}\left(q^{b}\right)=\sum_{\mathbf{k}}\left[\begin{array}{c}
b+a_{1} \\
k_{1}
\end{array}\right]\left[\begin{array}{c}
b+a_{2} \\
k_{2}
\end{array}\right] \ldots\left[\begin{array}{c}
b+a_{n} \\
k_{n}
\end{array}\right]_{x_{1}, \ldots, x_{n}}^{\mathrm{CT}} L^{\prime}\left(x_{1}, \ldots, x_{n}, \mathbf{a}, \mathbf{k}\right),
$$

where the sum ranges over all sequences $\mathbf{k}=\left(k_{1}, \ldots, k_{n}\right)$ of nonnegative integers such that $k_{1}+k_{2}+\cdots+k_{n}=a_{1}+a_{2}+\cdots+a_{n}$, and $L^{\prime}\left(x_{1}, \ldots, x_{n}, \mathbf{a}, \mathbf{k}\right)$ is a Laurent 
polynomial in $x_{1}, \ldots, x_{n}$ independent of $b$. Since $\left[\begin{array}{c}b+a_{i} \\ k_{i}\end{array}\right]$ is a polynomial in $q^{b}$ of degree $k_{i}$, each summand in (3.4) is a polynomial in $q^{b}$ of degree $k_{1}+k_{2}+\cdots+k_{n}=$ $a_{1}+a_{2}+\cdots+a_{n}$, and so is the sum.

Proof of Theorem 1.2. We proceed by induction on $n$. Theorem 1.2 is trivial for $n=0$ and reduces to the $q$-binomial theorem for $n=1$. Suppose the theorem is true for $n$ variables. We may call these variables $x_{1}, \ldots, x_{n}$ rather than $x_{0}, \ldots x_{n-1}$, so our induction hypothesis implies that (1.2) holds when $a_{0}=0$.

We will show that $P_{\mathbf{a}}\left(q^{a_{0}}\right)=Q_{\mathbf{a}}\left(q^{a_{0}}\right)$ for all nonnegative integers $a_{0}$. We know that (i) $P_{\mathbf{a}}\left(q^{0}\right)=Q_{\mathbf{a}}\left(q^{0}\right)$ by the induction hypothesis; (ii) by Lemma 3.1, both $P_{\mathbf{a}}\left(q^{b}\right)$ and $Q_{\mathbf{a}}\left(q^{b}\right)$ define polynomials in $q^{b}$ of degree no greater than $a$; (iii) by Lemma 3.2, $P_{\mathbf{a}}\left(q^{b}\right)=Q_{\mathbf{a}}\left(q^{b}\right)=0$ for $q^{b}=q^{-1}, q^{-2}, \ldots, q^{-a}$. So $P_{\mathbf{a}}\left(q^{b}\right)$ and $Q_{\mathbf{a}}\left(q^{b}\right)$ are equal as polynomials in $q^{b}$.

\section{Constant term evaluations}

We will evaluate the constant term $Q_{\mathbf{a}}\left(q^{b}\right)$ defined by (3.2), where $b$ is a negative integer, by partial fraction expansion. Although we are taking the constant term of a Laurent series in $x_{0}$ with coefficients that are Laurent polynomials in $x_{1}, \ldots, x_{n}$, when we expand by partial fractions, we get terms that are not of this form, and in order to evaluate their constant terms we need to work in a larger ring: the field of iterated Laurent series $K\left\langle\left\langle x_{n}, x_{n-1}, \ldots, x_{0}\right\rangle\right\rangle=K\left(\left(x_{n}\right)\right)\left(\left(x_{n-1}\right)\right) \ldots\left(\left(x_{0}\right)\right)$, where $K=\mathbb{C}(q)$, in which all series are regarded first as Laurent series in $x_{0}$, then as Laurent series in $x_{1}$, and so on. For a more detailed account of the properties of this field, with other applications, see [13] and [14.

Every element of $K\left\langle\left\langle x_{n}, x_{n-1}, \ldots, x_{0}\right\rangle\right\rangle$ has a unique Laurent series expansion. The series expansions of $1 /\left(1-q^{k} x_{i} / x_{j}\right)$ will be especially important. If $i<j$, then

$$
\frac{1}{1-q^{k} x_{i} / x_{j}}=\sum_{l=0}^{\infty} q^{k l} x_{i}^{l} x_{j}^{-l} .
$$

However, if $i>j$, then this expansion is not valid and instead we have the expansion

$$
\frac{1}{1-q^{k} x_{i} / x_{j}}=\frac{1}{-q^{k} x_{i} / x_{j}\left(1-q^{-k} x_{j} / x_{i}\right)}=\sum_{l=0}^{\infty}-q^{-k(l+1)} x_{i}^{-l-1} x_{j}^{l+1} .
$$

Let $F(\mathbf{x})$ be in $K\left\langle\left\langle x_{n}, x_{n-1}, \ldots, x_{0}\right\rangle\right\rangle$. The constant term of $F(\mathbf{x})$ in $x_{i}$, denoted by $\mathrm{CT}_{x_{i}} F(\mathbf{x})$, is defined to be the sum of those terms in the series expansion of $F(\mathbf{x})$ that are free of $x_{i}$. This definition clearly extends the constant term operators used earlier. It follows that

$$
\mathrm{CT}_{x_{i}} \frac{1}{1-q^{k} x_{i} / x_{j}}= \begin{cases}1, & \text { if } i<j \\ 0, & \text { if } i>j .\end{cases}
$$

We shall call the monomial $M=q^{k} x_{i} / x_{j}$ small if $i<j$ and large if $i>j$. Thus the constant term in $x_{i}$ of $1 /(1-M)$ is 1 if $M$ is small and 0 if $M$ is large.

An important property of the constant term operators defined in this way is their commutativity:

$$
\underset{x_{i}}{\mathrm{CT}} \underset{x_{j}}{\mathrm{CT}} F(\mathbf{x})=\underset{x_{j}}{\mathrm{CT}} \underset{x_{i}}{\mathrm{CT}} F(\mathbf{x}) .
$$

Commutativity implies that the constant term in a set of variables is well defined, and this property will be used in our proof of the Main Lemma. (Note that, by contrast, the constant term operators in [15] do not commute.) 
The degree of a rational function of $x$ is the degree in $x$ of the numerator minus the degree in $x$ of the denominator. For example, if $i \neq j$, then the degree of $1-x_{j} / x_{i}=\left(x_{i}-x_{j}\right) / x_{i}$ is 0 in $x_{i}$ and 1 in $x_{j}$. A rational function is called proper in $x$ if its degree in $x$ is negative. The following lemma gives a formula for the constant term in $x_{k}$ of certain elements of $K\left\langle\left\langle x_{n}, x_{n-1}, \ldots, x_{0}\right\rangle\right\rangle$ which are proper rational functions of $x_{k}$.

Lemma 4.1. Let

$$
R=\frac{p\left(x_{k}\right)}{x_{k}^{d} \prod_{i=1}^{m}\left(1-x_{k} / \alpha_{i}\right)}
$$

be a proper rational function of $x_{k}$, where $p\left(x_{k}\right)$ is a polynomial in $x_{k}$, and the $\alpha_{i}$ are distinct monomials, each of the form $x_{t} q^{s}$. Then

$$
\underset{x_{k}}{\mathrm{CT}} R=\left.\sum_{j}\left(R\left(1-x_{k} / \alpha_{j}\right)\right)\right|_{x_{k}=\alpha_{j}},
$$

where the sum ranges over all $j$ such that $x_{k} / \alpha_{j}$ is small.

Proof. The field $K\left\langle\left\langle x_{n}, \ldots, x_{0}\right\rangle\right\rangle$ contains the polynomial ring $K\left[x_{0}, \ldots, x_{n}\right]$ as a subring and hence contains the field $K\left(x_{0}, \ldots, x_{n}\right)$ of rational functions as a subfield. Thus any identity in $K\left(x_{0}, \ldots, x_{n}\right)$ is also an identity in $K\left\langle\left\langle x_{n}, \ldots, x_{0}\right\rangle\right\rangle$.

The partial fraction decomposition of $R$ with respect to $x_{k}$ is

$$
\frac{p\left(x_{k}\right)}{x_{k}^{d} \prod_{i=1}^{m}\left(1-x_{k} / \alpha_{i}\right)}=\frac{p_{0}\left(x_{k}\right)}{x_{k}^{d}}+\left.\sum_{j=1}^{m} \frac{1}{1-x_{k} / \alpha_{j}}\left(\frac{p\left(x_{k}\right)}{x_{k}^{d} \prod_{i=1, i \neq j}^{m}\left(1-x_{k} / \alpha_{i}\right)}\right)\right|_{x_{k}=\alpha_{j}},
$$

where $p_{0}\left(x_{k}\right)$ is a polynomial in $x_{k}$ of degree less than $d$. The term $p_{0}\left(x_{k}\right) / x_{k}^{d}$ contributes nothing to the constant term in $x_{k}$, and $1 /\left(1-x_{k} / \alpha_{j}\right)$ contributes to the constant term in $x_{k}$ only if $x_{k} / \alpha_{j}$ is small. The result of the lemma then follows easily.

The following lemma plays an important role in our argument.

Lemma 4.2. Let $A_{1}, \ldots, A_{s}$ be nonnegative integers. Then for any positive integers $k_{1}, \ldots, k_{s}$ with $1 \leq k_{i} \leq A_{1}+\cdots+A_{s}$ for all $i$, either $1 \leq k_{i} \leq A_{i}$ for some $i$ or $-A_{j} \leq k_{i}-k_{j} \leq \bar{A}_{i}-\overline{1}$ for some $i<j$.

Proof. We prove by contradiction that there is no $k_{1}, \ldots, k_{s}$ such that for all $i$, $A_{i}<k_{i} \leq A_{1}+\cdots+A_{s}$, and for all $i<j$, either $k_{i}-k_{j} \geq A_{i}$ or $k_{i}-k_{j} \leq-A_{j}-1$. Suppose $k_{1}, \ldots, k_{s}$ do satisfy these conditions. We construct a tournament on $1,2, \ldots, s$ with numbers on the arcs as follows: For $i<j$, if $k_{i}-k_{j} \geq A_{i}$, then we draw an arc $i \stackrel{A_{i}}{\longleftarrow} j$ from $j$ to $i$ and if $k_{i}-k_{j} \leq-1-A_{j}$, then we draw an arc $i \stackrel{A_{j}+1}{\longrightarrow} j$ from $i$ to $j$.

We call an arc from $u$ to $v$ an ascending arc if $u<v$ and a descending arc if $u>v$. We note two facts: (i) the number on an arc from $u$ to $v$ is less than or equal to $k_{v}-k_{u}$, and (ii) the number on an ascending arc is always positive.

A consequence of (i) is that for any directed path from $e$ to $f$, the sum along the arcs is less than or equal to $k_{f}-k_{e}$. It follows that the sum along a cycle is nonpositive. But any cycle must have at least one ascending arc, and by (ii) the number on this arc is positive, and so the sum along the cycle is positive. Thus there can be no cycles. 
Therefore the tournament we have constructed is transitive, and hence defines a total ordering $\rightarrow$ on $1,2, \ldots, s$. Assume the total ordering is given by $i_{1} \rightarrow i_{2} \rightarrow$ $\cdots \rightarrow i_{s-1} \rightarrow i_{s}$. Then $k_{i_{s}}-k_{i_{1}} \geq A_{i_{2}}+A_{i_{3}}+\cdots+A_{i_{s}}$. This implies that

$$
\begin{aligned}
k_{i_{s}} & \geq k_{i_{1}}+A_{i_{2}}+A_{i_{3}}+\cdots+A_{i_{s}} \\
& >A_{i_{1}}+A_{i_{2}}+A_{i_{3}}+\cdots+A_{i_{s}} \\
& =A_{1}+A_{2}+\cdots+A_{s},
\end{aligned}
$$

a contradiction.

\section{Proof of the Main Lemma}

Let

$$
\mathcal{Q}(b)=\prod_{j=1}^{n} \frac{\left(x_{j} q / x_{0}\right)_{a_{j}}}{\left(1-x_{0} / x_{j} q\right)\left(1-x_{0} / x_{j} q^{2}\right) \cdots\left(1-x_{0} / x_{j} q^{b}\right)} \prod_{1 \leq i<j \leq n}\left(\frac{x_{i}}{x_{j}}\right)_{a_{i}}\left(\frac{x_{j}}{x_{i}} q\right)_{a_{j}},
$$

so that in the notation of the previous section, $\mathrm{CT}_{\mathbf{x}} \mathcal{Q}(b)=Q_{\mathbf{a}}\left(q^{-b}\right)$. Our goal is to show that $\mathrm{CT}_{\mathbf{x}} \mathcal{Q}(b)=0$ for $b=1,2, \ldots, a$.

Since the degree in $x_{0}$ of $1-x_{j} q^{i} / x_{0}=\left(x_{0}-x_{j} q^{i}\right) / x_{0}$ is $0, \mathcal{Q}(b)$ is proper in $x_{0}$, with degree $-n b$.

Applying Lemma 4.1, we have

$$
\mathrm{CT}_{x_{0}} \mathcal{Q}(b)=\sum_{\substack{0<r_{1} \leq n \\ 1 \leq k_{1} \leq b}} \mathcal{Q}\left(b \mid r_{1} ; k_{1}\right)
$$

where

$$
\mathcal{Q}\left(b \mid r_{1} ; k_{1}\right)=\left.\mathcal{Q}(b)\left(1-\frac{x_{0}}{x_{r_{1}} q^{k_{1}}}\right)\right|_{x_{0}=x_{r_{1}} q^{k_{1}}} .
$$

For each term in (5.1) we will extract the constant term in $x_{r_{1}}$, and then perform further constant term extractions, eliminating one variable at each step. In order to keep track of the terms we obtain, we introduce some notation.

For any rational function $F$ of $x_{0}, x_{1}, \ldots, x_{n}$, and for sequences of integers $\mathbf{k}=$ $\left(k_{1}, k_{2}, \ldots, k_{s}\right)$ and $\mathbf{r}=\left(r_{1}, r_{2}, \ldots, r_{s}\right)$, let $E_{\mathbf{r}, \mathbf{k}} F$ be the result of replacing $x_{r_{i}}$ in $F$ with $x_{r_{s}} q^{k_{s}-k_{i}}$ for $i=0,1, \ldots, s-1$, where we set $r_{0}=k_{0}=0$. Then for $0<r_{1}<r_{2}<\cdots<r_{s} \leq n$ and $0<k_{i} \leq b$, we define

$$
\mathcal{Q}(b \mid \mathbf{r} ; \mathbf{k})=\mathcal{Q}\left(b \mid r_{1}, \ldots, r_{s} ; k_{1}, \ldots, k_{s}\right)=E_{\mathbf{r}, \mathbf{k}}\left[\mathcal{Q}(b) \prod_{i=1}^{s}\left(1-\frac{x_{0}}{x_{r_{i}} q^{k_{i}}}\right)\right] .
$$

Note that the product on the right-hand side of (5.2) cancels all the factors in the denominator of $\mathcal{Q}$ that would be taken to zero by $E_{\mathbf{r}, \mathbf{k}}$.

Lemma 5.1. The rational functions $\mathcal{Q}(b \mid \mathbf{r} ; \mathbf{k})$ have the following two properties:

(i) If $1 \leq k_{i} \leq a_{r_{1}}+\cdots+a_{r_{s}}$ for all $i$ with $1 \leq i \leq s$, then $\mathcal{Q}(b \mid \mathbf{r} ; \mathbf{k})=0$.

(ii) If $k_{i}>a_{r_{1}}+\cdots+a_{r_{s}}$ for some $i$ with $1 \leq i \leq s$ and $n>s$, then

$$
\underset{x_{s}}{\mathrm{CT}} \mathcal{Q}(b \mid \mathbf{r} ; \mathbf{k})=\sum_{\substack{r_{s}<r_{s+1} \leq n \\ 1 \leq k_{s+1} \leq b}} \mathcal{Q}\left(b \mid r_{1}, \ldots, r_{s}, r_{s+1} ; k_{1}, \ldots, k_{s}, k_{s+1}\right) .
$$


Proof of property (i). By Lemma4.2, either $1 \leq k_{i} \leq a_{r_{i}}$ for some $i$ with $1 \leq i \leq s$, or $-a_{r_{j}} \leq k_{i}-k_{j} \leq a_{r_{i}}-1$ for some $i<j$. If $1 \leq k_{i} \leq a_{r_{i}}$, then $\mathcal{Q}(b \mid \mathbf{r} ; \mathbf{k})$ has the factor

$$
E_{\mathbf{r}, \mathbf{k}}\left[\left(\frac{x_{r_{i}}}{x_{0}} q\right)_{a_{r_{i}}}\right]=\left(\frac{x_{r_{s}} q^{k_{s}-k_{i}}}{x_{r_{s}} q^{k_{s}}} q\right)_{a_{r_{i}}}=\left(q^{1-k_{i}}\right)_{a_{r_{i}}}=0 .
$$

If $-a_{r_{j}} \leq k_{i}-k_{j} \leq a_{r_{i}}-1$ where $i<j$, then $\mathcal{Q}(b \mid \mathbf{r} ; \mathbf{k})$ has the factor

$$
E_{\mathbf{r}, \mathbf{k}}\left[\left(\frac{x_{r_{i}}}{x_{r_{j}}}\right)_{a_{r_{i}}}\left(\frac{x_{r_{j}}}{x_{r_{i}}} q\right)_{a_{r_{j}}}\right]
$$

which by (2.6) is equal to

$$
\begin{aligned}
& q^{\left(a_{r_{2}}^{a_{2}+1}\right)}\left(-\frac{x_{r_{j}}}{x_{r_{i}}}\right)^{a_{r_{j}}}\left(\frac{x_{r_{i}}}{x_{r_{j}}} q^{-a_{r_{j}}}\right)_{a_{r_{i}}+a_{r_{j}}} \\
& \left.=q^{\left(a_{r_{j}}+1\right.}\right)\left(-q^{k_{i}-k_{j}}\right)^{a_{r_{j}}}\left(q^{k_{j}-k_{i}-a_{r_{j}}}\right)_{a_{r_{i}}+a_{r_{j}}}=0 .
\end{aligned}
$$

Proof of property (ii). Note that since $b \geq k_{i}$ for all $i$, the hypothesis implies that $b>a_{r_{1}}+\cdots+a_{r_{s}}$.

We first show that $\mathcal{Q}(b \mid \mathbf{r} ; \mathbf{k})$ is proper in $x_{r_{s}}$. To do this we write $\mathcal{Q}(b \mid \mathbf{r} ; \mathbf{k})$ as $N / D$, in which $N$ (the "numerator") is

$$
E_{\mathbf{r}, \mathbf{k}}\left[\prod_{j=1}^{n}\left(\frac{x_{j}}{x_{0}} q\right)_{a_{j}} \cdot \prod_{\substack{1 \leq i, j \leq n \\ j \neq i}}\left(\frac{x_{i}}{x_{j}} q^{\chi(i>j)}\right)_{a_{i}}\right],
$$

where $\chi(S)$ is 1 if the statement $S$ is true, and 0 otherwise, and $D$ (the "denominator") is

$$
E_{\mathbf{r}, \mathbf{k}}\left[\prod_{j=1}^{n}\left(\frac{x_{0}}{x_{j} q^{b}}\right)_{b} / \prod_{i=1}^{s}\left(1-\frac{x_{0}}{x_{r_{i}} q^{k_{i}}}\right)\right] .
$$

Now let $R=\left\{r_{0}, r_{1}, \ldots, r_{s}\right\}$. Then the degree in $x_{s}$ of

$$
E_{\mathbf{r}, \mathbf{k}}\left[\left(1-\frac{x_{i}}{x_{j}} q^{l}\right)\right]
$$

is 1 if $i \in R$ and $j \notin R$, and is 0 otherwise, as is easily seen by checking the four cases. Thus the part of $N$ contributing to the degree in $x_{r_{s}}$ is

$$
E_{\mathbf{r}, \mathbf{k}}\left[\prod_{i=1}^{s} \prod_{j \neq r_{0}, \ldots, r_{s}}\left(\frac{x_{r_{i}}}{x_{j}} q^{\chi\left(r_{i}>j\right)}\right)_{a_{r_{i}}}\right],
$$

which has degree $(n-s)\left(a_{r_{1}}+\cdots+a_{r_{s}}\right)$, and the part of $D$ contributing to the degree in $x_{r_{s}}$ is

$$
E_{\mathbf{r}, \mathbf{k}}\left[\prod_{j \neq r_{0}, \ldots, r_{s}}\left(\frac{x_{0}}{x_{j} q^{b}}\right)_{b}\right],
$$

which has degree $(n-s) b$.

Thus the total degree of $\mathcal{Q}(b \mid \mathbf{r} ; \mathbf{k})$ in $x_{r_{s}}$ is $(n-s)\left(a_{r_{1}}+\cdots+a_{r_{s}}-b\right)<0$, so $\mathcal{Q}(b \mid \mathbf{r} ; \mathbf{k})$ is proper in $x_{r_{s}}$. 
Next we apply Lemma 4.1. For any rational function $F$ of $x_{r_{s}}$ and integers $j$ and $k$, let $T_{j, k} F$ be the result of replacing $x_{r_{s}}$ with $x_{j} q^{k-k_{s}}$ in $F$. Since $x_{r_{s}} q^{k_{s}} /\left(x_{j} q^{k}\right)$ is small when $j>r_{s}$ and is large when $j<r_{s}$, Lemma 4.1 gives

$$
\underset{x_{s}}{\mathrm{CT}} \mathcal{Q}(b \mid \mathbf{r} ; \mathbf{k})=\sum_{\substack{r_{s}<r_{s+1} \leq n \\ 1 \leq k_{s+1} \leq b}} T_{r_{s+1}, k_{s+1}}\left[\mathcal{Q}(b \mid \mathbf{r} ; \mathbf{k})\left(1-\frac{x_{r_{s}} q^{k_{s}}}{x_{r_{s+1}} q^{k_{s+1}}}\right)\right] .
$$

We must show that the right-hand side of (5.4) is equal to the right-hand side of (5.3). Let us set $\mathbf{r}^{\prime}=\left(r_{1}, \ldots, r_{s}, r_{s+1}\right)$ and $\mathbf{k}^{\prime}=\left(k_{1}, \ldots, k_{s}, k_{s+1}\right)$. Then the equality follows easily from the identity

$$
T_{r_{s+1}, k_{s+1}} \circ E_{\mathbf{r}, \mathbf{k}}=E_{\mathbf{r}^{\prime}, \mathbf{k}^{\prime}} .
$$

To see that (5.5) holds, we have

$$
\left(T_{r_{s+1}, k_{s+1}} \circ E_{\mathbf{r}, \mathbf{k}}\right) x_{r_{i}}=T_{r_{s+1}, k_{s+1}}\left[x_{r_{s}} q^{k_{s}-k_{i}}\right]=x_{r_{s+1}} q^{k_{s+1}-k_{i}}=E_{\mathbf{r}^{\prime}, \mathbf{k}^{\prime}} x_{r_{i}},
$$

and if $j \notin\left\{r_{0}, \ldots, r_{s}\right\}$, then $\left(T_{r_{s+1}, k_{s+1}} \circ E_{\mathbf{r}, \mathbf{k}}\right) x_{j}=x_{j}=E_{\mathbf{r}^{\prime}, \mathbf{k}^{\prime}} x_{j}$.

Proof of the Main Lemma. We prove by induction on $n-s$ that

$$
\mathrm{CT}_{\mathbf{x}} \mathcal{Q}(b \mid \mathbf{r} ; \mathbf{k})=0
$$

the Main Lemma is the case $s=0$. (Note that taking the constant term with respect to a variable that does not appear has no effect.)

We may assume that $s \leq n$ and $0<r_{1}<\cdots<r_{s} \leq n$, since otherwise $\mathcal{Q}(b \mid \mathbf{r} ; \mathbf{k})$ is not defined. If $s=n$, then $r_{i}$ must equal $i$ for $i=1, \ldots, n$ and thus $\mathcal{Q}(b \mid \mathbf{r} ; \mathbf{k})=\mathcal{Q}\left(b \mid 1,2, \ldots, n ; k_{1}, k_{2}, \ldots, k_{n}\right)$, which is 0 by part (i) of Lemma 5.1 . since $k_{i} \leq b \leq a_{1}+\cdots+a_{n}$ for each $i$.

Now suppose that $0 \leq s<n$. If part (i) of Lemma 5.1]applies, then $\mathcal{Q}(b \mid \mathbf{r} ; \mathbf{k})=0$. Otherwise, part (ii) of Lemma 5.1 applies, and (5.3) holds. Applying $\mathrm{CT}_{\mathbf{x}}$ to both sides of (5.3) gives

$$
\mathrm{CT} \mathcal{Q}(b \mid \mathbf{r} ; \mathbf{k})=\sum_{\substack{r_{s}<r_{s+1} \leq n \\ 1 \leq k_{s+1} \leq b}} \operatorname{CT}_{\mathbf{x}} \mathcal{Q}\left(b \mid r_{1}, \ldots, r_{s}, r_{s+1} ; k_{1}, \ldots, k_{s}, k_{s+1}\right),
$$

and by induction, every term on the right is 0 .

\section{REFERENCES}

1. G. E. Andrews, Problems and prospects for basic hypergeometric functions, in Theory and Application of Special Functions, ed. R. A. Askey, Academic Press, New York, 1975, pp. 191224. MR0399528 (53:3372)

2. - The Theory of Partitions, Encyclopedia of Mathematics and its Applications, Vol. 2, Addison-Wesley, Reading, Mass., 1976. MR0557013 (58:27738)

3. D. M. Bressoud and I. P. Goulden, Constant term identities extending the $q$-Dyson theorem, Trans. Amer. Math. Soc. 291 (1985), 203-228. MR0797055 (86k:05011)

4. I. Cherednik, Double affine Hecke algebras and Macdonald's conjectures, Ann. of Math. (2) 141 (1995), 191-216. MR1314036 (96m:33010)

5. I. J. Good, Short proof of a conjecture by Dyson, J. Math. Phys. 11 (1970), 1884. MR0258644 $(41: 3290)$

6. J. Gunson, Proof of a conjecture by Dyson in the statistical theory of energy levels, J. Math. Phys. 3 (1962), 752-753. MR0148401 (26:5908)

7. K. W. J. Kadell, A proof of Andrews' $q$-Dyson conjecture for $n=4$, Trans. Amer. Math. Soc. 290 (1985), 127-144. MR0787958 (86f:33003)

8. I. G. Macdonald, Some conjectures for root systems SIAM J. Math. Anal. 13 (1982), 9881007. MR0674768 (84h:17006a) 
9. R. P. Stanley, The q-Dyson conjecture, generalized exponents, and the internal product of Schur functions, Combinatorics and algebra (Boulder, Colo., 1983), Contemp. Math., vol. 34, Amer. Math. Soc., Providence, RI, 1984, pp. 81-94. MR0777696 (86i:22026)

10. $\quad$ The stable behavior of some characters of $\mathrm{SL}(n, \mathbf{C})$, Linear and Multilinear Algebra 16 (1984), 3-27. MR0768993(86e:22025)

11. J. R. Stembridge, A short proof of Macdonald's conjecture for the root systems of type A, Proc. Amer. Math. Soc. 102 (1988), 777-786. MR0934842 (89e:11062)

12. K. G. Wilson, Proof of a conjecture by Dyson, J. Math. Phys. 3 (1962), 1040-1043. MR $0144627(26: 2170)$

13. G. Xin, A residue theorem for Malcev-Neumann series, Adv. in Appl. Math. 35 (2005), 271293. MR 2164920

14. , A fast algorithm for MacMahon's partition analysis, Electron. J. Combin. 11 (2004), 20 pp. MR2097324

15. D. Zeilberger, Proof of a conjecture of Chan, Robbins, and Yuen, Elec. Trans. Numer. Anal. 9 (1999), 147-148. MR1749805 (2001b:05015)

16. D. Zeilberger and D. M. Bressoud, A proof of Andrews' q-Dyson conjecture, Discrete Math. 54 (1985), 201-224. MR0791661 (87f:05015)

Department of Mathematics, Brandeis University, Waltham, Massachusetts 024549110

E-mail address: gessel@brandeis.edu

Department of Mathematics, Brandeis University, Waltham Massachusetts 024549110

E-mail address: guoce.xin@gmail.com 